\title{
Strength criterion for unidirectional CFRP under off-axis loading
}

\author{
Alexander Dumansky ${ }^{1,2, *}$, Hao Liu $^{2}$, and Mukhamiat Alimov ${ }^{1}$ \\ ${ }^{1}$ Blagonravov Mechanical Engineering Research Institite of the Russian Academy of Sciences, \\ Materials Sciences Department, 4 Maly Kharitonyevsky Pereulok, Moscow, 101990, Russia \\ ${ }^{2}$ Bauman Moscow State Technical University, Production Department, 5/1 $2^{\text {nd }}$ Baumanskaya Street, \\ Moscow, 105005, Russia
}

\begin{abstract}
A failure criterion is proposed which allows describing the strength of unidirectional CFRP under tension-compression at an off-axis angle with strength applicable for practical use. An experimental verification was carried out which showed satisfactory agreement with the experimental results. The criterion makes it possible to determine the peak of the failure envelope in the compression area caused, on the one hand, by an increase in strength under in-plane shear due to transverse compressive stresses and, on the other hand, by a decrease in strength due to destruction of the matrix and the interface between unidirectional layer components.
\end{abstract}

\section{Introduction}

Most of the works devoted to the development of models of mechanical behavior are based on the unidirectional layer properties [1-3]. Depending on the goal of the problem, the regularities are revealed for one or another type of loading and failure. The behavior of components of thin-walled structures in the first approximation is based on the relations of the theory of layer elasticity and the basic principles of the theory of layered plates. The most complete models of the mechanical behavior of polymer composites under quasi-static loading are presented in [1]. In recent years, there has been an increase in the number of publications related to the study of the effect of strain rate and other experiments showing the rheological properties of unidirectional composites [4]. At the same time, special emphasis is placed on the study of the regularities of mechanical behavior under dynamic and impact loading. In this paper, using unidirectional carbon fiber reinforced plastic AS4/3501-6 as an example, a failure criterion is considered which can be extended to include the effect of strain rate. The data for testing the proposed criterion were digitized from [5]. The review [6] analyzed the phenomenological criteria for the strength of anisotropic materials, the geometry of fracture surfaces, and experimental methods for determining these criteria. A comparison was made and the features of the criteria were noted, in particular, the criteria of maximum stresses and strains, tensor polynomial criteria, formulated as polynomials in stresses or strains. Criteria for maximum values are not interactive and do not take into account the mutual influence of the components on the ultimate state of a material.

*Corresponding author: aldumans@rambler.ru 
Criteria of the tensor polynomial form in which the main difficulty is the determination of the coefficients of mutual influence of components are considered interactive. As a rule, these components include the values of normal stresses while the relationship between normal and shear stresses is not considered. The effect of mutual influence of normal and shear stresses as an experimental fact, along with other features, was noted in a number of works [4, 5, 714]. In particular, a strength criterion [14] was proposed which, along with the strength values and dimensionless parameters depending on elastic characteristics, makes it possible to evaluate the dependence of strength on the direction of loading and, as a consequence, allows describing the peak of in-plane shear strength depending on the level of transverse compressive stresses. The formulated strength conditions are associated with the corresponding types of fracture. It is assumed that when the extremum is passed, a change in the type of fracture is observed.

\section{Tensor polynomial criteria}

The strength anisotropy of unidirectional composites can be described using phenomenological failure criteria based on the strength characteristics determined from test results both in the direction of the principal axes of orthotropy and at different off-axis angles as well as under combined stress state. The degree of adequacy of the criterion is the agreement between experimental and calculated data, including the features of mechanical behavior of material. Criteria of tensor polynomial type allow considering the mutual influence of stress components on strength. In particular, the frequently used tensorpolynomial Tsai-Wu failure criterion is the sum of linear and quadratic parts and has the following form:

$$
F_{i} \sigma_{i}+F_{i j} \sigma_{i} \sigma_{j}=1 \text {, }
$$

where the components of criterion (1) are determined from the results of uniaxial tensioncompression tests, and the components $\mathrm{F}_{\mathrm{ij}}$, when $\mathrm{i} \neq \mathrm{j}$, allow taking into account the mutual influence of the stress components and are determined based on the condition of minimizing the discrepancy between experimental and calculated data. The closure condition of the fracture surface follows from the condition that the quadratic form of the criterion is positive definite and imposes the following restriction on the components of the criterion: $\mathrm{F}_{\mathrm{ii}} \mathrm{F}_{\mathrm{jj}}-\mathrm{F}_{\mathrm{ij}}{ }^{2}>0$. It follows from the latter that the parameter for assessing the mutual influence of the components is in the range $-\sqrt{F_{i i} F_{j j}}<F_{i j}<\sqrt{F_{i i} F_{j j}}$.

The tensor polynomial strength criterion for a two-dimensional stress state, in the general case, takes the following form [6]

$$
F_{11} \sigma_{1}^{2}+F_{22} \sigma_{2}^{2}+F_{66} \sigma_{6}^{2}+2 F_{12} \sigma_{1} \sigma_{2}+2 F_{16} \sigma_{1} \sigma_{6}+2 F_{26} \sigma_{2} \sigma_{6}+F_{1} \sigma_{1}+F_{2} \sigma_{2}+F_{6} \sigma_{6}=1,
$$

where $F_{i}=\frac{1}{\sigma_{i}^{+}}-\frac{1}{\sigma_{i}^{-}}, F_{i i}=\frac{1}{\sigma_{i}^{+} \sigma_{i}^{-}}, \sigma_{i}^{ \pm}$are the tensile and compressive strength values. In [6], the types of surfaces and the relations for determining the parameters of criterion (2) are discussed. It was stated that $F_{16}=F_{26}=0$. The conditions for determining $F_{12}$ are stipulated in a large number of works. It should be noted that in case of off-axis loading of a unidirectional composite at which fracture occurs in the form of delamination, criterion (2) is transformed to the following form

$$
F_{22} \sigma_{2}^{2}+F_{66} \sigma_{6}^{2}+F_{2} \sigma_{2+} F_{6} \sigma_{6}=1
$$

In [12], when a matrix of unidirectional composite being destroyed, the following criterion was considered 


$$
\left(\frac{1}{\sigma_{2}^{+}}-\frac{1}{\sigma_{2}^{-}}\right) \sigma_{2}+\frac{\sigma_{2}^{2}}{\sigma_{2}^{+} \sigma_{2}^{-}}+\frac{\tau_{6}^{2}}{\tau_{6}^{* 2}}=1
$$

The test results for unidirectional CFRP specimens calculated using criterion (4) are shown in Figure 1. From Figure 1, it can be seen that the failure envelope is an ellipse in the principal axes with a shift along the axis $\sigma_{2}$. The numerical values of the parameters were determined using experimental data.

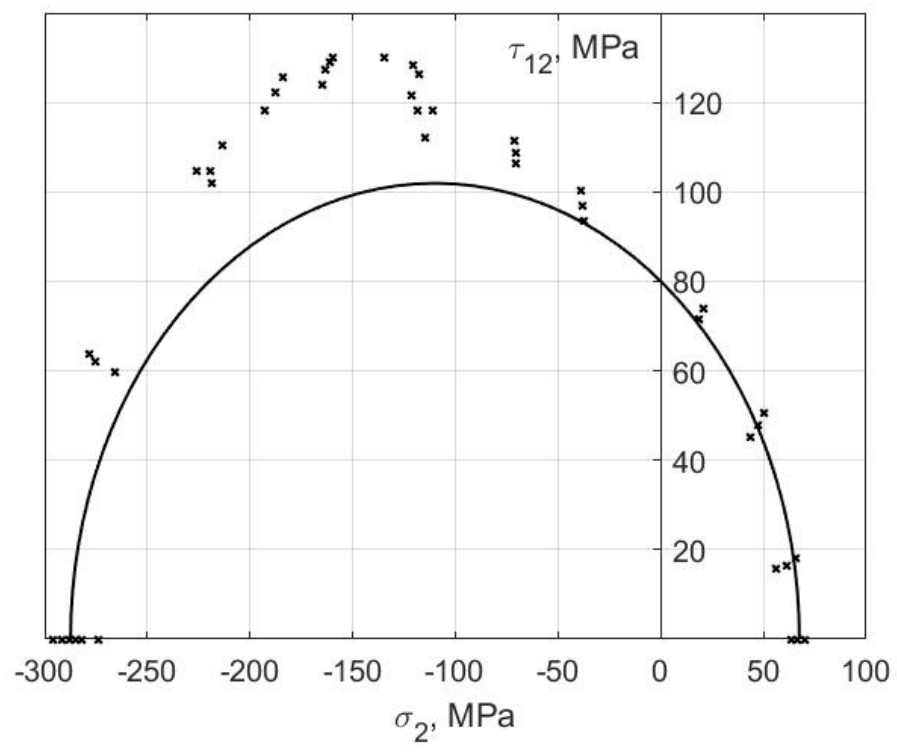

Fig. 1. Comparison of the calculated and experimental data for AS4/3501-6 composite.

It can be seen from Figure 1 that criterion (4) is in good agreement with the experiment in case of tension and compression in the direction of reinforcement, but, in area of compression at angles not equal to $90^{\circ}$, the calculation differs distinctly from experiment.

It should be noted that it is assumed in the criteria for maximum stresses and tensorpolynomial criteria that there is no mutual influence of normal stresses and shear, so the test results are worse than for criterion (4). However, the test results for some composites, especially CFRPs, show the presence of such a relationship, namely, a noticeable effect of tension or compression in the direction perpendicular to the reinforcement on the in-plane shear strength. The presence of tensile stresses during shear obviously reduces the strength of a unidirectional composite and satisfactorily fits into the corresponding $\sigma_{2}-\tau_{12}$ quadrant using the Hashin's quadratic criterion, and a peak of strength is observed experimentally during compression. The effect of compressive stresses on shear strength could be taken into account by adding the corresponding terms to the failure criterion. The above effect was described in [4-10]. In [14], the strength peak was explained by a change in the nature of fracture associated with the predominance of fracture from shear or from compression. At the same time, dimensionless parameters related to the moduli of elasticity under shear and loading in the direction perpendicular to the reinforcement were introduced into the criterion estimates. If we assume that the properties of the material follow the law of elasticity, then the force criteria can be related in a certain way to deformation ones. In [15], the value of the parameter characterizing the degree of friction in the presence of transverse compressive stresses was experimentally determined. The Sun criterion is as follows 


$$
\left(\frac{\sigma_{2}}{\sigma_{2}^{-}}\right)^{2}+\left(\frac{\tau_{6}}{\tau_{6}^{*}-\eta \sigma_{2}}\right)^{2}=1
$$

where $\eta$ is an experimentally determined parameter that is given the meaning of internal friction.

\section{Model description}

This paper is based on the analysis of experimental data from the results of testing specimens under quasi-static off-axis loading of specimens made of AS4/3501-6 carbon fiber reinforced plastic. Experimental data were digitized from figures in [4]. A failure criterion is proposed in the form of a parabolic curve

$$
\tau_{6}=\mathrm{a} \sigma_{2}^{2}+b \sigma_{2}+c
$$

The parabola described by equation (5) can be constructed in two possible ways. The first way to construct a curve by the least squares method in which the discrepancy between the experimental and calculated data is minimized. The resulting equation looks like this

$$
\tau_{6}=-0.0037 \sigma_{2}^{2}-0.8528 \sigma_{2}+81.4
$$

The roots of equation (6) should be the calculated values of the compressive and tensile strength, and the point of intersection of the parabola with the ordinate $\tau_{6}$ should be the value of the pure shear strength, i.e., in the absence of transverse stresses. The calculated transverse strength values turned out to be equal to $\sigma_{2}^{+}=72.4 \mathrm{MPa}$ for tension and $\sigma_{2}^{-}=299.8 \mathrm{MPa}$ for compression. The calculated in-plane shear strength value of is equal to the free term in the parabola equation, i.e. $\tau_{6}^{*}=81.4 \mathrm{MPa}$.

A parabolic relationship can also be constructed using three points that can be taken from experimental data. In particular, from [4]: $\sigma_{2}^{+}=65 \mathrm{MPa}, \sigma_{2}^{-}=285 \mathrm{MPa}$, and $\tau_{6}^{*}=80 \mathrm{MPa}$. The parabola equation passing through three characteristic points: $\left(-\sigma_{2}^{-}, 0\right),\left(0, \tau_{6}^{*}\right),\left(\sigma_{2}^{+}\right)$ can be constructed by solving the following system of linear equations

$$
\left(\begin{array}{ccc}
\left(\sigma_{2}^{-}\right)^{2} & \sigma_{2}^{-} & 1 \\
0 & 0 & 1 \\
\left(\sigma_{2}^{+}\right)^{2} & \sigma_{2}^{+} & 1
\end{array}\right)\left(\begin{array}{l}
a \\
b \\
c
\end{array}\right)=\left(\begin{array}{c}
0 \\
\tau_{6}^{*} \\
0
\end{array}\right)
$$

In this case, the parabola equation has the following form

$$
\tau_{6}=-\frac{\tau_{6}^{*}}{\sigma_{2}^{+} \sigma_{2}^{-}} \sigma_{2}^{2}-\tau_{6}^{*}\left(\frac{1}{\sigma_{2}^{+}}-\frac{1}{\sigma_{2}^{-}}\right) \sigma_{2}+\tau_{6}^{*}
$$

The numerical value of the parabola strength obtained from the experimental values turned out to be

$$
\tau_{6}=-0.0041 \sigma_{2}^{2}-0.9068 \sigma_{2}+80
$$

A comparison of the experimental points and the results of calculations based on dependencies (3) and (5) is shown in Figure 2. It can be noted that using the known parameters of the parabola, the maximum in-plane shear strength value and the transverse stress value corresponding to the maximum shear strength can be found. For the empirical curve of the parabola, we have $\sigma_{2 s y m}=-110 \mathrm{MPa}$ and $\tau_{6 \max }=132,5 \mathrm{MPa}$, and for the parabola the parameters of which were determined by the least squares method, we have $\sigma_{2 \text { sym }}=-113.7 \mathrm{MPa}$ and $\tau_{6 \max }=129,9 \mathrm{MPa}$. It should be noted that for the failure envelope determined using the criterion proposed in [5], the experimental points, except for 
the basic ones, lie below the failure envelope. Criterion (4) can be reduced to the following form

$$
\left(\frac{1}{\sigma_{2}^{+}}-\frac{1}{\sigma_{2}^{-}}\right) \sigma_{2}+\frac{\sigma_{2}^{2}}{\sigma_{2}^{+} \sigma_{2}^{-}}+\frac{\tau_{6}}{\tau_{6}^{*}}=1
$$

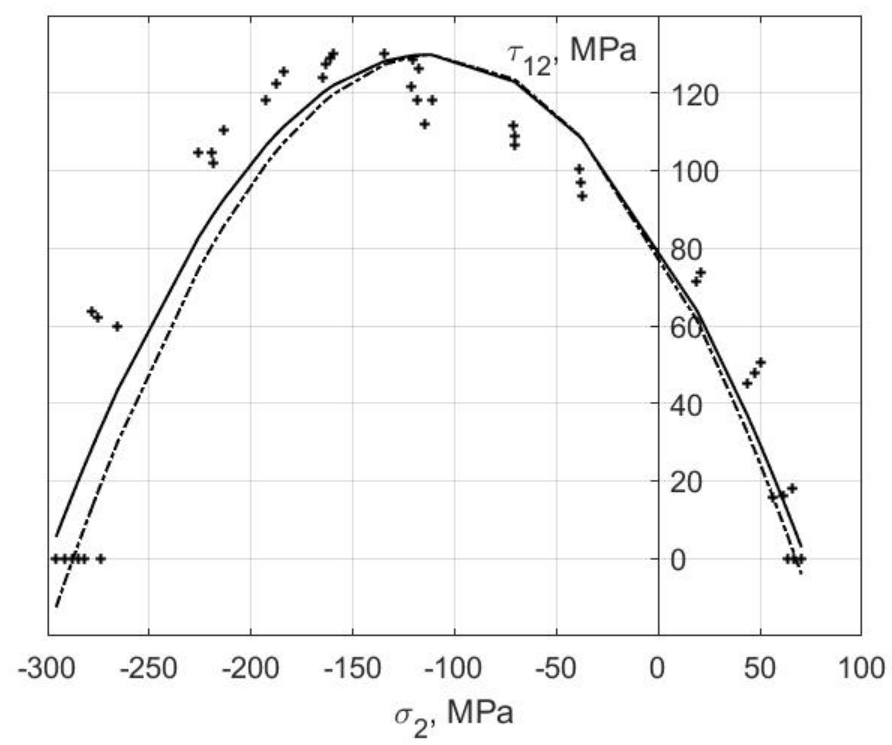

Fig. 2. Comparison of calculated and experimental strength data on strength for AS4/3501-6 CFRP specimens under quasi-static loading. Crosses - experimental data, solid line - calculation by the least squares method, dashed line - calculation by equation (8).

For comparison, Table 1 shows the experimental and calculated values of the failure criterion parameters.

Table 1. Failure criterion parameters.

\begin{tabular}{|c|c|c|c|}
\hline & $\boldsymbol{\sigma}_{2}^{+}, \mathbf{M P a}$ & $\boldsymbol{\sigma}_{\mathbf{2}}^{-}, \mathbf{M P a}$ & $\boldsymbol{\tau}_{\mathbf{6}}^{*}, \mathbf{M P a}$ \\
\hline Experiment & 65 & 285 & 80 \\
\hline $\begin{array}{c}\text { Calculation } \\
\text { (LSM) }\end{array}$ & 72,4 & 299,8 & 81,4 \\
\hline $\begin{array}{c}\text { Calculation } \\
\text { (control } \\
\text { points) }\end{array}$ & 67.5 & 287.4 & 80 \\
\hline
\end{tabular}

To check the correlation between the calculated and experimental data, the coefficient of determination was calculated for the curve constructed by the method of least squares and for the parabolic curve constructed by using the experimental results. In the latter case, the value of the shear strength equal to $80 \mathrm{MPa}$ is taken from the experimental data. The $R-$ squared coefficient was calculated using the following relation

$$
R^{2}=1-\frac{\sum\left(\tau_{6 i}^{e x p}-\tau_{6 i}^{c a l c}\right)^{2}}{\sum\left(\tau_{6 i}^{e x p}-\tau_{6}^{a v e}\right)^{2}}
$$

where $\tau_{6}^{a v e}$ is the average of all values of intra-layer strength 
For the values calculated by the least squares method, the $R$ - squared coefficient turned out to be 0.9028 , and for the values obtained by using the experimental data $K_{d}=0.8792$. It can be seen that the obtained difference is small, the LSM calculation turned out to be somewhat higher, however, in the first and second cases, it can be concluded that the calculated and experimental data are in satisfactory agreement. The scattering of the data obtained by the LSM as a whole is somewhat better suited to the experimental data. Note that, by virtue of the accepted conditions, the parabola constructed on the basis of the experimental points accurately describes the results of tests under tension-compression in the transverse direction and in-plane shear.

\section{Conclusion}

The method for assessing the strength of unidirectional CFRP under off-axis loading is proposed. To describe the strength, the parabolic dependence of the in-plane shear strength on the transverse stresses was used. The parameters of the curve were determined by the least squares method as well as by using the control points of the parabola. The performed analysis showed the possibility of using both methods, and the least squares method more accurately describes the experimental data, however, it allows some error in the control points. The parabolic dependence of the strength built from the proposed points has generally less accuracy but accurately describes the tensile-compressive strength in the transverse direction.

\section{References}

1. Failure Criteria in Fibre Reinforced Polymer Composites: The World-Wide failure Exercise, Eds: M.J. Hinton, A.S. Kaddiur, P.D. Soden, (2004)

2. P.D. Soden, A.S. Kaddour, M.J. Hinton, Compos. Sci. Technol., 64, (2004)

3. C.T. Herakovich, Mechanics of Fibrous Composites, (1998)

4. P.P. Camanho, A. Arteiro, A.R. Melro, G. Catalanotti, M. Vogler, Intern. J. Solids and Struct. (2014)

5. I.M. Daniel, B.T. Werner, J.S. Fenner, Compos. Sci. and Tech, 71 (2011).

6. E. Wu in: L. J. Broutman and R. H. Krock (eds.), Composite Materials. Vol. 2. G. P. Sendeckyj (ed.), Mechanics of Composite Materials, Academic Press, New York-London (1974)

7. C. Schuecker and H. E. Pettermann, In: Mechanical Response of Composites P.P. Camanho, C.G. Davila, S.T. Pinho, J.J.C. Remmers Eds. (2008)

8. M. Knops, Analysis of Failure in Fiber Polymer Laminates The Theory of Alfred Puck (2008)

9. Puck A, Schurmann H Compos Sci Technol 58, pp. 1045-1067 (1998)

10. H. Koerber, P.P. Xavier, P.P. Camanho, Mechanics of Materials, pp. 1004-1019 (2010).

11. R.M. Kristensen, S.J. DeTeresa, J. Compos. Mats, 38 (2004)

12. R.M. Kristensen, S.J. DeTeresa, Int.J.Solids Struct, 40,(2003)

13. H. Koerber, P. Kuhn, M. Ploekl, F. Otero, P-W. Gerbaud, R. Rolfes, P.P. Camanho Adv. Model. and Simul. In Eng. Sci, pp. 5-17 (2018)

14. I. M. Daniel, Y-J. Luo, P.M. Schubel, B.T. Werner Composite Sci. and Technology, 69 $(2009$

15. C.G. Davila, P.P. Camanho, C.A. Rose, J. Compos. Mater. 39, v. 4, (2005) 